\title{
Necesidad de tratamiento periodontal en adultos diabéticos controlados y no controlados en una población chilena. Estudio de corte transversal
}

\author{
Need for periodontal treatment among adults with controlled \\ and uncontrolled diabetes in a Chilean population. \\ A cross sectional study
}

\author{
Gutiérrez $\mathrm{MF}^{1}$, Hernández $\mathrm{JP}^{1}$, Juárez $\mathrm{IA}^{2}$, Aravena $\mathrm{P}^{3}$
}

\begin{abstract}
RESUMEN
Objetivo: Observar Necesidad de Tratamiento Periodontal (NTP) en pacientes adultos sin Diabetes Mellitus tipo 2 (noDM2) y con Diabetes Mellitus tipo 2 (DM2) controlados y no controlados en una población chilena. Materiales y Métodos: Estudio observacional de corte transversal. Un periodoncista registró la NTP según el Índice Comunitario de Necesidad de Tratamiento Periodontal (CPITN), en una muestra de pacientes noDM2, DM2 controlados (Hemoglobina Glicosilada HbA1c $\leq$ a 6.5\%) y DM2 no controlados (HbA1c > 6.5\%) del Centro Clínico Militar de Valdivia-Chile entre abril y julio del 2012. Se registró edad, género, y presencia de hipertensión arterial. Se analizaron las variables sociodemográficas y la asociación estadística entre NTP en pacientes noDM2, con DM2 controlados y no controlados (test chi-cuadrado, $p<0.05$ ). Resultados: Noventa y seis pacientes fueron analizados con una edad promedio de $55.2 \pm 8.3$ años de los cuales $45(48.9 \%)$ eran diabéticos. El $96.88 \%$ de la muestra presentaron una NTP por parte de especialista (códigos 3 y 4 del CPITN), no existiendo diferencia significativa entre pacientes DM2 y noDM2 ( $p=0.63$ ). No se observaron diferencias significativas en NTP entre aquellos pacientes con diabetes controlada y no controlada ( $p=0.43$ ). Conclusiones: Reportes previos relacionan la presencia y la falta de control de DM2 como factor de riesgo de enfermedad periodontal. Sin embargo, en nuestro estudio existió un alto índice de NTP especializada en todos los tipos de pacientes examinados.
\end{abstract}

Rev. Clin. Periodoncia Implantol. Rehabil. Oral Vol. 6(2); 67-70, 2013.

Palabras clave: Periodontitis, diabetes mellitus, hemoglobina glucosilada, índice comunitario de necesidad de tratamiento periodontal, Chile.

\section{ABSTRACT}

Objective: To observe the Periodontal Treatment Need (PTN) in adult patients without Diabetes Mellitus type 2 (noDM2) and with Diabetes Mellitus type 2 (DM2) controlled and non-controlled in a chilean population. Materials and Methods: Cross sectional study. A periodontist recorded the PTN according to the Periodontal Treatment Need Community Index (CPITN), from a sample of patients with noDM2, controlled DM2 (Hemoglobin A, Glycosylated $\mathrm{HbA} 1 \mathrm{c} \leq \mathrm{a} 6.5 \%)$ and non-controlled DM2 ( $\mathrm{HbA} 1 \mathrm{c}>6.5 \%)$ from the Army Clinical Center in Valdivia-Chile between april and july 2012. The age, sex and presence of arterial hypertension were recorded. The sociodemographic variables and the statistical association between PTN in patients with noDM2, with controlled and non-controlled DM2 (chi-squared test, $p<0.05$ ) were also analyzed. Results: Ninety six patients were analyzed. The average age was $55.2 \pm 8.3$ years old, 45 of them $(48.9 \%)$ were diabetics. $96.88 \%$ of the samples presented a PTN by the specialists (CPITN codes 3 and 4), there were no significant differences between DM2 and noDM2 patients ( $p=0.63$ ). No significant differences were observed in PTN between patients with controlled and non-controlled diabetes $(p=0.43)$. Conclusions: Previous reports related the presence and lack of control of DM2 as a risk factor of periodontal disease. Nonetheless, in our study, there was a high index of PTN in all the different types of patients examined.

Rev. Clin. Periodoncia Implantol. Rehabil. Oral Vol. 6(2); 67-70, 2013.

Key words: Periodontitis, diabetes mellitus, periodontal index, hemoglobin A, glycosylated, Chile.

\section{INTRODUCCIÓN}

La Diabetes Mellitus es una enfermedad crónica de alta prevalencia, la cual, ha aumentado progresivamente en las últimas décadas $^{(1)}$. En Chile, actualmente la prevalencia de Diabetes Mellitus tipo 2 (DM2) es de 9.4\%. Por otro lado, la enfermedad periodontal (EP) tiene una prevalencia de $98.8 \%$ en adultos entre $35-44$ años y de $100 \%$ en adultos entre 65-74 años ${ }^{(2)}$.

Se estima que la prevalencia de periodontitis en pacientes diabéticos es el doble o el triple, en comparación con personas no diabéticas $^{(3)}$ y un cuadro de DM2 pobremente contralada es asociado con mayor prevalencia y severidad de periodontitis ${ }^{(4)}$, a tal punto que se

1. Cirujano Dentista. Escuela de Odontología. Facultad de Medicina, Universidad Austral de Chile. Chile.

2. Cirujano Dentista. Especialista en Periodoncia. Profesor Adjunto. Escuela de Odontología. Facultad de Medicina, Universidad Austral de Chile. Chile.

3. Cirujano Dentista. Profesor Instructor. Escuela de Odontología. Facultad de Medicina, Universidad Austral de Chile. Chile.

Correspondencia autor: Israel Juárez Membreño. juarezuach@gmail.com. Trabajo recibido el 25/10/2012. Aprobado para su publicación el 04/03/2013. 
considera un factor de riesgo para el desarrollo de la enfermedad(5).

Existen posturas que no asocian la relación entre el nivel de Hemoglobina glucosilada y la severidad de la $\mathrm{EP}^{(6,7)}$ como también que no existe asociación entre periodontitis e incidencia de diabetes $^{(8)}$

A la fecha, no hay estudios disponibles en base de datos electrónicas que hayan demostrado la relación entre presencia ni control de DM2 y necesidad de tratamiento periodontal (NTP) en una población chilena, siendo perentorio su registro para comprobar su real asociación a nivel local.

El objetivo del estudio es observar la NTP en pacientes adultos atendidos en el Centro Clínico Militar de la Ciudad de Valdivia-Chile sin Diabetes Mellitus tipo 2 (noDM2) y con Diabetes Mellitus tipo 2 (DM2) controlados y no controlados.

\section{MATERIALES Y MÉTODOS}

Se diseñó un estudio de corte transversal. Se obtuvo una muestra de pacientes atendidos en el Centro Clínico Militar de la ciudad de Valdivia entre los meses de enero del año 2011 y julio del año 2012 previa aprobación por parte del comité de ética y gestión de dicho centro de salud.

Para obtener la muestra de pacientes con DM2 se utilizó un método no probabilístico consecutivo a partir de una lista de atención de los servicios de medicina general y medicina interna del año 2011. Los criterios de inclusión fueron: personas diagnosticadas con diabetes mellitus tipo 2, personas mayores de 20 años, con al menos dos dientes en boca y que aceptaron participar mediante la firma de consentimiento informado. Fueron excluidos pacientes con tratamiento periodontal previo en los últimos 6 meses, tratamiento periodontal actual, embarazadas, pacientes diagnosticados con otras patologías crónicas como hipotiroidismo, hipertiroidismo, asma, enfermedad pulmonar obstrucitva crónica, insuficiencia renal crónica, pacientes diagnosticados con alguna patología cardiovascular a excepción de la hipertensión arterial y pacientes fumadores moderados (6-15 cigarros al día) y severos (más de 16 cigarrillos al día) ${ }^{(9)}$.

De los pacientes seleccionados, se revisaron las fichas médicas para corroborar que existía el diagnóstico de la enfermedad bajo los criterios de la Asociación Latinoamericana de Diabetes ${ }^{(10)}$ y conocer el resultado del último examen de hemoglobina glucosilada (HbA1c) registrada, como máximo, ocho semanas después del examen clínico, considerando el tiempo durante el cual el nivel de hemoglobina glucosilada se mantiene estable ${ }^{(11)}$. Todos los exámenes de $\mathrm{HbA1c}$ fueron tomados con un instrumento marca Abbott, modelo Axsym $\mathrm{HbA} 1 \mathrm{c}$, y a través de la técnica MEIA (enzimoinmunoensayo con micropartícula o autoanalizador inmunológico) perteneciente al Laboratorio Clínico del Centro Clínico Militar de Valdivia.

Para el grupo de sujetos noDM2 se escogieron pacientes atendidos en el centro clínico durante el año 2011 en medicina general y medicina interna, a través de muestreo aleatorio estratificado según la edad obtenida del grupo de pacientes con DM2. Fueron revisadas las fichas clínicas y dentales de todos los individuos durante los meses de marzo a junio. Los pacientes seleccionados fueron invitados a participar del examen periodontal vía telefónica y mediante una invitación escrita.

Tanto al grupo de pacientes noDM2 y DM2, un periodoncista (IJ) regristró el Índice Comunitario de necesidad de tratamiento periodontal(12) utilizando una sonda tipo IPC de la OMS marca Hu-friedy en dos tiempos clínicos con una semana de diferencia, considerando el registro de datos como idóneos con un índice kappa en las mediciones igual o sobre a un índice 0.8 .

Se utilizaron códigos de puntuación que van del 0 al $4^{(12)}$. Los terceros molares sólo se consideraron si reemplazaban la función del segundo molar. El sextante con un solo diente se registró como faltante (código $X$ ) y este diente se incluyó en el sector adyacente. Se excluyeron los dientes con indicación de exodoncia. Para cada sextante, se asignó un código según el valor más alto encontrado en el sondaje periodontal.

La evaluación fue realizada en un tiempo de 15 minutos y los resultados fueron registrados en una ficha de recolección de datos.
Pacientes con necesidad de tratamiento por un especialista fueron derivados a tratamiento dentro del mismo establecimiento.

En cada grupo de pacientes (DM2 y noDM2) fueron registradas las siguientes variables: Sexo (Femenino/ Masculino), edad distribuída en cuatro grupos (desde 20 a 44 años, 45 a 64 años, 65 a 74 años y sobre 75 años). En pacientes con DM2 se clasificó su estado de control de diabetes según el índice de hemoglobina glicosilada en: DM2 controlados $(\mathrm{HbA} 1 \mathrm{c} \leq \mathrm{a} 6.5 \%)$ y no controlados $(\mathrm{HbA} 1 \mathrm{c}>6.5 \%)^{(11)}$ Como variable dependiente se consideró la NTP clasificando los códigos obtenidos en dos grupos:

- Código 0, 1 y 2: Sin NTP por especialista.

- Código 3 y 4: NTP por especialista.

Para determinar el tamaño de la muestra se consideró una prevalencia esperada de $87 \%$ de pacientes con NTP realizado por un especialista ${ }^{(13)}$, un nivel de confianza del $95 \%$ y un margen de error aceptado para la población real de $7 \%$ obteniendo un cálculo de 51 pacientes para cada grupo de estudio.

La codificación de los datos fue en forma numérica en un formulario de Google Docs. El ingreso de los datos obtenidos fue realizado por uno de los autores (MG). Se analizó una asociación estadistica entre el tipo de paciente (noDM2 y DM2), el estado de control de DM2 y la NTP mediante test chi-cuadrado con un nivel de significancia $p<0.05$. Tanto el cálculo de tamaño muestral, la tabulación y el manejo de los datos fue realizado con el software estadístico SPSS Statistics versión 17.0 (SPSS Inc., Chicago, EE.UU.).

\section{RESULTADOS}

340 registros clínicos cumplieron los criterios de selección, de los cuales 102 pacientes atendieron el llamado y fueron examinados por el especialista. En total, se obtuvo una muestra de 96 pacientes, 49 hombres y 53 mujeres con un promedio de edad de $55.2 \pm 8.3$ años. El proceso de selección y el seguimiento de pacientes se describen en la Figura 1.

Del total de la muestra, $45(44.11 \%)$ pertenecieron al grupo DM2 y 51 al grupo no DM2, no observándose diferencias estadísticamente significativas según género $(p=0.1)$ ni edad $(p=0.22)$ entre ambos grupos. Sin embargo, se observó una diferencia estadísticamente significativa en la presencia de HTA en el grupo DM2 $(p=0.0006)$ (Tabla 1$)$.

En forma adicional, se analizó la presencia de códigos $X$ por sextante en cada individuo observándose una mayor cantidad de sextantes edentulos en el grupo DM2 en comparación con el grupo noDM2 (Figura 2). La presencia de códigos $x$ en pacientes diabéticos es significativamente estadístico en comparación con pacientes no diabéticos en los sextantes $1(p=0.01)$ y en el sextante $4(p=0.03)$.

En el examen periodontal se observa un $96.88 \%$ de toda la muestra estudiada tiene necesidad NTP por parte de un especialista, no exisitiendo diferencia significativa entre pacientes del grupo noDM2 y DM2 ( $p=0.63$ ) (Tabla 2$)$.

Existe un $62.22 \%$ de pacientes con DM2 que presenta una diabetes no controlada según el examen $\mathrm{HbA} 1 \mathrm{c}$. No se observaron diferencias significativas en la necesidad de tratamiento periodontal entre pacientes con diabetes controlada y no controlada $(p=0.43)$ (Tabla 3). 


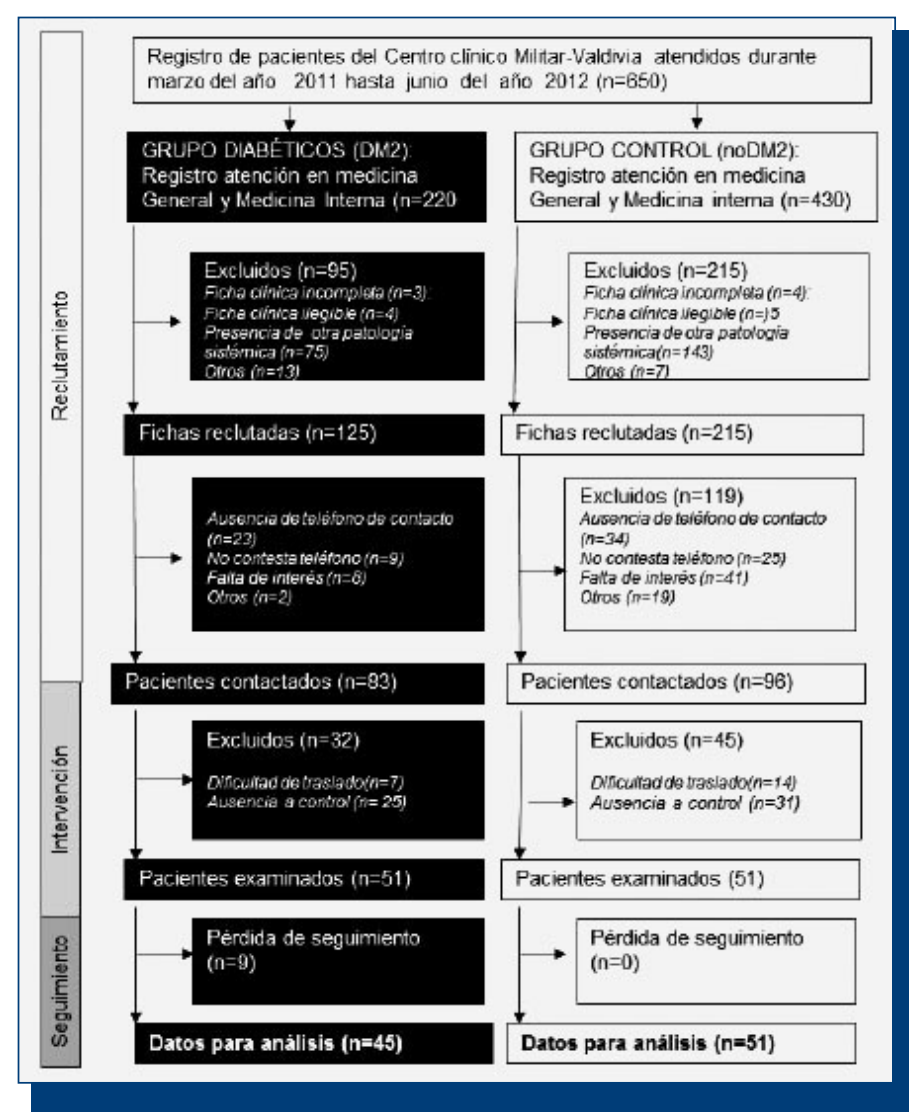

Figura 1. Flujograma de reclutamiento, selección y seguimiento de pacientes por grupo de estudio.

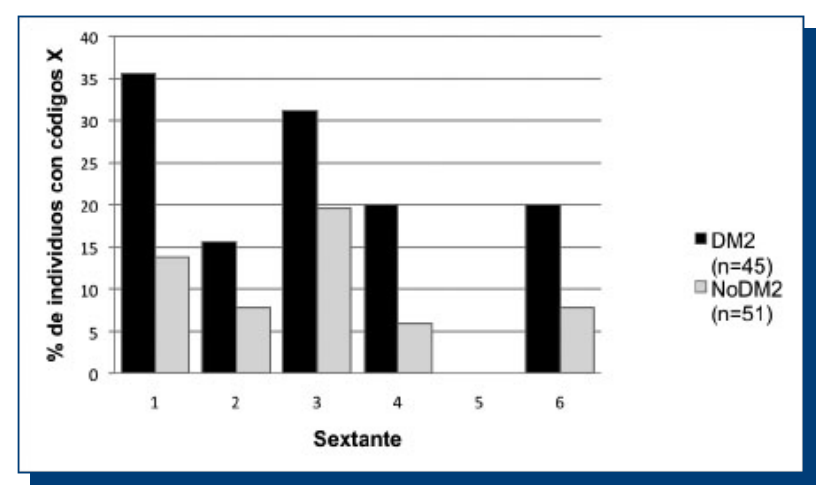

Figura 2. Distribución porcentual de individuos con códigos $X$ de CPITN (sextantes edéntulos), entre DM2 y noDM2 por sextante.

\section{DISCUSIÓN}

En nuestro estudio se observó una alta frecuencia de NTP a realizar por un especialista en periodoncia tanto en pacientes no DM2 y DM2 controlados y no controlados. Además, la presencia de códigos " $X$ " fue mayor en el grupo de pacientes con DM2, lo que puede sugerir una mayor pérdida dentaria en este grupo de pacientes.

El hecho de realizar el estudio en una población restringida solamente a personas que tienen la previsión del ejército no permite conocer la realidad de NTP de quienes se atienden en el sistema público u otros centros de atención. Otra limitación es la elección de un punto de corte para las medidas de hemoglobina A1c. Por la dicotomización de hemoglobina A1c, los sujetos con valores ligeramente por encima o por debajo de la línea de corte tienden a ser similares con respecto
Tabla 1. Características sociodemográficas de diabéticos (DM2) y grupo control (noDM2) de la muestra de estudio.

\begin{tabular}{|c|c|c|c|}
\hline & DM2 ( $n=45)$ & noDM2 ( $\mathrm{n}=51)$ & \\
\hline & $\mathbf{N}^{0}(\%)$ & № (\%) & $p^{1}$ \\
\hline Sexo & & & 0.10 \\
\hline Masculino & $19(42.22)$ & $30(58.82)$ & \\
\hline Femenino & $26(57.78)$ & $21(41.18)$ & \\
\hline Edad & & & 0.22 \\
\hline $24-44$ & $2(4.44)$ & $5(9.8)$ & \\
\hline $45-64$ & $38(84.44)$ & $45(88.24)$ & \\
\hline $65-74$ & $4(8.89)$ & $1(1.96)$ & \\
\hline 75 y más & $1(2.22)$ & $0(0)$ & \\
\hline \multicolumn{4}{|l|}{ HbA1c } \\
\hline Controlada $(\mathrm{HbA} 1 \mathrm{c} \leq \mathrm{a} 6.5 \%)$ & $17(37.78)$ & - & \\
\hline No controlada ( $\mathrm{HbA} 1 \mathrm{C}>6.5 \%)$ & $28(62.22)$ & - & \\
\hline HTA & & & $0.0006^{*}$ \\
\hline Presencia & $28(62.22)$ & $14(27.45)$ & \\
\hline Ausencia & $17(37.78)$ & $37(72.55)$ & \\
\hline
\end{tabular}

1. Prueba chi cuadrado $(\mathrm{p}<0.05)$

* Significancia estadística.

Tabla 2. Distribución de necesidad de tratamiento periodontal (NTP) realizado por un especialista según grupo de DM2 y noDM2.

\begin{tabular}{|lll|}
\hline \hline No de pacientes por grupo & Con NTP (\%) & Sin NTP (\%) \\
\hline DM2 & $44(97.78)$ & $1(2.22)$ \\
\hline noDM2 & $49(96.08)$ & $2(3.92)$ \\
\hline Total & $93(96.88)$ & $3(3.12)$ \\
\hline
\end{tabular}

Tabla 3. Distribución de necesidad de tratamiento periodontal (NTP) realizado por un especialista en pacientes con DM2 según control glucémico medido a través de valores de hemoglobina glucosilada (HbA1c).

\begin{tabular}{|lcc|}
\hline \hline No de pacientes en control de DM2 & Con NTP (\%) & Sin NTP (\%) \\
\hline Controlada $(\mathrm{HbA1c} \leq \mathrm{a} 6.5 \%)$ & $17(100)$ & $0(0)$ \\
\hline No controlada $(\mathrm{HbA} 1 \mathrm{c}>6.5 \%)$ & $27(96.43)$ & $1(3.57)$ \\
\hline Total & $44(97.77)$ & $1(2.22)$ \\
\hline
\end{tabular}

a la situación de control de glucemia, aunque clasificados como una exposición diferente en el análisis.

Cuando se compara la NTP en códigos 3 y 4 y condición de DM2 con reportes previos de Linares-Vieyra $C$ et al. ${ }^{(14)}$ y Martha $B$ et al. ${ }^{(15)}$, se puede observar que han registrado una frecuencia similar en la NTP de $90.1 \%$ y $96.8 \%$, respectivamente. Sin embargo, estos estudios presentan diferencias metodológicas importantes como son la definición de diabetes, la falta de especificación sobre la cantidad de cigarrillos diarios necesarios para la clasificación de fumador y la falta de exclusión de pacientes con otras enfermedades crónicas. Incluso investigaciones previas $^{(4,6)}$, utilizan distintos valores de corte de hemoglobina glucosilada y usan como herramienta de control glucémico en DM2 el examen de glucemia en ayunas ${ }^{(14-16)}$. Sin embargo, se sostiene según los manejos clínicos internacionales actuales, que el examen de $\mathrm{HbA} 1 \mathrm{c}$ es el método más efectivo para determinar el control glucémico(11,17) tal como fue reportado en este estudio.

Al analizar solamente el escenario de NTP complejo (que incluye códigos 3 y 4) y la frecuencia observada en pacientes con DM2 se refleja un alto porcentaje de pacientes con posible infección periodontal compleja. Un cuadro de hiperglucemia en un paciente diabético influye en el desarrollo de infecciones, generado por el desbalance entre la acción y secreción de insulina como también el aumento de productos 
finales en la glucosilación avanzada (AGEs) los que ocasionan en los tejidos un aumento del estrés oxidativo y una alteración significativa de la composición y estructura celular normal de macrofagos/monocitos, linfocitos, fibroblastos y células endoteliales. Estas células secretan mediadores los cuales finalmente tienen sus efectos locales en el sitio de inflamación, pero que repercute de forma sistémica y afectan la evolución de la DM y de sus complicaciones crónicas ${ }^{(18-20)}$, alterando el control metabólico ${ }^{(19,20)}$, su nivel de calidad y duración ${ }^{(18)}$. La presencia de un pobre control de DM, medida a través de los niveles plasmáticos de hemoglobina glucosilada, se asocia con mayor prevalencia, severidad y extensión de la enfermedad periodontal ${ }^{(18,21-23)}$ y cambios asociados con alteraciones celulares como la disminución de producción de colágeno por el fibroblasto ${ }^{(24)}$, que generan anormalidades en la cicatrización, alteraciones microvasculares ${ }^{(25,26)}$, incremento de susceptibilidad a infecciones entre ellas periodontales y alteraciones en el metabolismo óseo ${ }^{(25)}$ que implica mayor producción de metaloproteinasas de la matriz ${ }^{(26)}$.

Por otro lado, es importante considerar que la relación entre el alto índice NTP y la presencia de DM2 podría orientar al dentista a la pesquisa de diabetes en aquellos pacientes que no son diagnosticados como tal ${ }^{(27)}$ o que tienen un estado de hiperglucemia ${ }^{(26)}$, hiperlipidemia(28) en una patología crónica, puesto que la presencia de sacos periodontales profundos y pérdida de 4 o más dientes permitiría identificar cerca de un $73 \%$ de casos de pacientes diabéticos ${ }^{(29)}$. Por lo tanto, se propone que la identificación de pacientes con NTP complejo puede ser un elemento clínico relevante para sospechar de DM no diagnosticada, y además un tratamiento adecuado de enfermedad periodontal, podría lograr un efecto positivo sobre el control metabólico de DM, el estado de inflamación circulatorio, actuando esto a la vez como elemento clave sobre la modulación de la enfermedad periodontal y la respuesta reparativa en los tejidos ${ }^{(20,30,31)}$.

Sin embargo, los datos obtenidos en la población estudiada no permiten afirmar lo propuesto anteriormente debido a la alta frecuencia de NTP complejo observada en toda la muestra analizada. Por lo tanto, en poblaciones donde la prevalencia de enfermedad periodontal es muy alta, el estado periodontal (representada por el grado de NTP) no permite distinguir la presencia de DM2. Por ello, es perentorio proponer exámenes periodontales rutinarios con mayor frecuencia, control glucémico y metabólico ${ }^{(31)}$ y disminuir el intervalo de tiempo de controles odontológicos en pacientes diabéticos y no diabéticos.

Según los datos obtenidos, no existirian diferencias significativas de NTP entre pacientes diabéticos y no diabéticos, más bien se presentó un alto índice de NTP por un especialista en todas las personas examinadas. Se propone realizar estudios observacionales mediante muestreo probabilístico a nivel nacional, diseño de estudios tipo analítico y experimentales con el fin de conocer cabalmente la asociación entre DM2 controlada y no controlada con la severidad de la EP.

Finalmente es importante un manejo interdisciplinario médicoodontológico de ambas patologías, promover la necesidad de la detección temprana y cerrar uniones entre los profesionales dentales y médicos en el manejo del paciente diabético.

\section{AGRADECIMIENTOS}

Agradecemos al los funcionarios del Centro Clínico Militar de Valdivia: Su Director, el Comandante Santiago Warnken Urzúa; a Subdirector Dental, Dr. Carlos Jacque Falcón; a los técnicos y encargados de Laboratorio Clínico y al Departamento de Fichas Clínicas.

\section{FINANCIAMIENTO Y CONFLICTO DE INTERESES}

Este estudio fue realizado en el Centro Clínico Militar de Valdivia quienes facilitaron las dependencias, el instrumental y el personal de apoyo, cualquier otro gasto monetario fue autofinanciado por los autores.

Los autores declaran no tener conflictos de interés.

\section{REFERENCIAS BIBLIOGRÁFICAS}

1. WHO. World Health Organization. Ginebra, Suiza: WHO, 2011

2. Gamonal J, López N, Aranda W. Periodontal conditions and treatment needs, by CPITN, in the 35-44 and 65-74 year-old population in Santiago, Chile. Int Dent $J$ 1998; 48(2): 96-103

3. Mealey B, Oates T. Diabetes Mellitus and periodontal diseases. J Periodontol 2006; 77(8): 1289-1303.

4. Tsai C, Hayes C, Taylor G. Glycemic control of type 2 diabetes and severe periodontal disease in the US adult population. Community Dent Oral Epidemiol, 2002; 30(3): 182-192.

5. Hussain A. The relationship between diabetes mellitus and periodontitis. Al Ameen J Med Sci, 2011; 4(1): 84-88.

6 . Tervonen T, Oliver R. Long term control of diabetes mellitus and periodontitis. $J$ Clin Periodontol, 1993; 20(6): 431-435.

7. Karikoski A, Murtomaa $\mathrm{H}$, llanne-Parikka P. Assessment of periodontal treatment needs among adults with diabetes in Finland. Int Dent J, 2002; 52(2): 75-80.

8. I de R, Hoshuyama T, Wilson D, Takahashi K, Higashi T. Periodontal disease and incident diabetes: A seven-year study. J Dent Res, 2011; 90(1): 41-46.

9. WHO. World Health Organization. Ginebra, Suiza: WHO, 2003.

10. Asociación Latinoamericana de Diabetes (ALAD). Guías ALAD de diagnóstico, control y tratamiento de la diabetes mellitus tipo 2. Comité de la Revista ALAD, 2006.

11. American Diabetes Association. Standards of medical care in diabetes. Diabetes Care, 2012; 35(1): 11-63

12. Ainamo J, Barmes D, Beagrie G, Cutress T, Martin J, Sardo-Infirri J. Development of the World Health Organization (WHO) community periodontal index of treatment needs (CPITN). Int Dent J, 1982; 32(3): 281-291.

13. Bakhshandeh S, Murtomaa H, Mofid R, Vehkalahti MM, Suomalainen K. Periodontal treatment needs of diabetic adults. J Clin Periodontol, 2007; 34(1): 537.

14. Linares-Vieyra C, González-Guevara MB, Rodríguez de Mendoza LE. Periodontal diseases and treatment needs in diabetes type 2. Rev Med Inst Mex Seguro Soc, 2009; 47(2): 129-134.

15. Martha B, González-Guevara MB, Linares-Vieyra C, Rodríguez de Mendoza LE. Prevalencia de trastornos bucales en población con diabetes mellitus tipo 2 . Rev Med Inst Mex Seguro Soc, 2008; 46(3): 237-245.

16. Ribeiro C, Ribeiro A, Átilas N, Abreu F. Evaluación periodontal de pacientes portadores de diabetes mellitus. Rev Cubana Estomatol, [revista en la Internet]. 2006 Jun [citado 2012 Oct 20]; 4 3(2). Disponible en: http://scielo.sld.cu/scielo. php?script=sci arttext\&pid=S0034-75072006000200004\&Ing=es.

17. Murray $A$, Jiménez $M$. Calidad del control glicémico según la hemoglobina glicosilada vs la glicemia en ayunas: Análisis en una población urbana y otra rura de diabéticos costarricenses. AMC, 2004; 46(3): 139-144
18. Juárez IA, Juárez XE, Carlo CV. Relación entre las enfermedades periodontales de la cavidad oral y el control glucémico en diabetes mellitus. Revista $A L A D, 2009$ 17(4): 128-138.

19. Taylor GW, Borgnakke WS. Periodontal disease: Associations with diabetes, glycemic control and complications. Oral Diseases, 2008; 14(3): 191-203.

20. Smith P, Retamal I, Cáceres M, Romero A, Silva D, Arancibia R, Martínez C. Diabetes y su impacto en el territorio periodontal. Rev Clin Periodoncia Implantol Rehabil Oral, 2012; 5(2): 90-92.

21. Loe H. Periodontal disease. The sixth complication of diabetes. Diabetes Care 1993; 16(1): 329-334.

22. Moles DR. Evidence of an association between diabetes and severity of periodontal disease. Evid Based Dent, 2006; 7(2): 45.

23. Khader YS, Dauod AS, El-Qaderi SS, Alkafajei A, Batayha WQ. Periodontal status of diabetics compared with nondiabetics: A meta-analysis. J Diabetes Complications, 2006; 20(1): 59-68

24. Soskolne W, Klinger A. The relationship between periodontal diseases and diabetes: An overview. Ann Periodontol, 2001; 6(1): 91-98.

25. Lalla E, Lambster IB, Schmidt AM. Enhanced interaction of advanced glycation end products with their cellular receptor RAGE: Implications for the pathogenesis of accelerated periodontal disease in diabetes. Ann Periodontol, 1998; 3(1): 1319.

26. Botero J, Yepes F, Roldán N, Castrillón C, Hincapie J, Ochoa S, Ospina C Becerra M, Jaramillo A, Gutiérrez S, Contreras A. Tooth and periodontal clinical attachment loss are associated with hyperglycemia in patients with diabetes. $J$ Periodontol, 2012; 83: 1245-1250.

27. National estimates and general information on diabetes and prediabetes in the United States. GA: US Department of Health and Human Services, Centers for Disease Control and Prevention. Atlanta. 2010.

28. Sangwan A, Tewari S, Singh H, Sharma R, Narula S. Periodontal status and hyperlipidemia: Statin users versus non-users. J Periodontol, 2013; 84: 3-12.

29. Lalla E, Kunzel C, Burkett S, Cheng B, Lamster IB. Identification of unrecognized diabetes and pre-diabetes in a dental setting. J Dent Res, 2011; 90(7): 855-860.

30. Chen L, Luo G, Xuan D, Wei B, Liu F, Li J, Zhan J. Effects of non-surgical periodontal treatment on clinical response, serum inflammatory parameters, and metabolic control in patiens with type 2 diabetes: A randomized study. $J$ Periodontol, 2012; 83: 435-443.

31. Juárez I, Juárez X, Caneppa G, Pérez M. Diabetes mellitus. Repercusión sobre el periodonto de la cavidad oral del ser humano. Revista $A L A D, 2008 ; 16$ (1): 26-33. 